\title{
DIAGNÓSTICO DE LA SITUACIÓN ACTUAL DEL EMPRENDIMIENTO EN LAS INSTITUCIONES EDUCATIVAS (GRADOS DECIMO Y ONCE) DE LA CIUDAD DE PASTO
}

\author{
Alicia Cristina Silva Calpa ${ }^{1}$ \\ Carlos Rubén Trejos Moncayo²
}

Recibido: 15 de mayo de 2013

Aceptado: 12 de agosto de 2013

\section{Resumen}

El presente artículo es el resultado de una investigación adelantada con el fin de diagnosticar la situación actual del emprendimiento, el entorno en el que se desarrolla y como los actores sociales participan en la formación de la cultura emprendedora de los estudiantes de décimo y once de las principales Instituciones Educativas de la ciudad de Pasto.

En primer lugar se estudiaron los factores claves de los Bachilleres que le permiten desarrollar una mentalidad emprendedora y así modelar su Perfil Emprendedor; Se elaboró una encuesta con el fin de conocer las aptitudes, actitudes, competencias y demás características que definen el perfil de los emprendedores en la cual se escoge aleatoriamente a los encuestados, al mismo tiempo se hace una búsqueda de los estudiantes que se han caracterizado por ser líderes en sus respectivos colegios para comparar información y resultados. Con los productos de la encuesta elaborada se determinaron los factores determinantes de éxito en construcción de una mentalidad emprendedora. Por último se analiza el entorno a través de la entrevista personalizada de una serie de actores que intervienen directa e indirectamente en el desarrollo y formación de la mentalidad emprendedora de los estudiantes de grado décimo y once de las instituciones educativas objeto de estudio.

Palabras claves: Emprendimiento social, educación media, cultura de emprendimiento, empresa.

\footnotetext{
${ }^{1}$ Administradora de Empresas, Especialista en Pedagogía para el Desarrollo del Aprendizaje Autónomo, Magister en Sistemas de Calidad y Productividad, Estudiante de Doctorado en Administración - UNAD Florida. Docente Escuela Ciencias Administrativas, Contables, Económicas y de Negocios, Investigadora principal Grupo Sindamanoy, líneas de investigación: emprendimiento, responsabilidad social y cadenas productivas. Correo electrónico alicia.silva@unad.edu.co C.C. No. 59823146 de Pasto Cel. 3157359556

${ }^{2}$ Administrador de Empresas, Especialista en Gerencia Estratégica de Mercadeo, Magíster en Mercadeo Agroindustrial. Empresario, Docente de la Escuela de Ciencias Administrativas Contables económicas y de Negocios ECACEN de la UNAD, investigador grupo Sindamanoy, líneas de investigación: emprendimiento y cadenas productivas. Correo electrónico carlos.trejos@unad.edu.co C.C. No. 98.394 .180 de Pasto Cel. 3165354186
} 


\title{
DIAGNOSIS OF THE CURRENT SITUATION OF ENTREPRENEURSHIP IN EDUCATIONAL INSTITUTIONS ( TEN AND ELEVEN COURSES) OF THE PASTO CITY
}

\begin{abstract}
The present document is an investigation realized in order to diagnose the current situation of the Entrepreneur, the environment in the one that develops and as the social actors they take part in the formation of the enterprising culture of the students of tenth and eleven of the principal Educational Institutions of the Pasto City.

In First there are studied the key factors of the Graduates who allow him to develop an enterprising and like that mentality to shape your Enterprising Profile; from this I elaborate a survey in order to know the aptitudes, attitudes, competences and other characteristics that define the profile of the enterprising potentials in which the polled ones be chosen at random, but at the same time there is done a search of the students who have been characterized for being leaders in your respective colleges to compare information and results. With the products of the elaborated survey the determinant factors of success decided in construction of an enterprising mentality. Finally the environment is analyzed across the personalized interview of a series of actors who intervene directly and indirectly in the development and formation of the enterprising mentality of the students of tenth degree and eleven of the educational institutions object of study.
\end{abstract}

Keywords: Social entrepreneurship, education media, culture of entrepreneurship, business.

\section{Introducción}

El grupo investigador busca diagnosticar la situación actual del emprendimiento, el entorno en el que se desarrolla y cómo los actores sociales participan en la formación de la cultura emprendedora y «cultura empresarial para la vida» (Muria, 2011:19) de los estudiantes de las Instituciones Educativas de la ciudad de Pasto.

Se asume como población objeto de estudio a los escolares de décimo y onceavo grado de las 7 principales instituciones educativas que albergan el mayor porcentaje de la población estudiantil de la ciudad de Pasto, población que requiere la efectiva intervención de la universidad a través de procesos de innovación conducentes a mejorar e incentivar condiciones competitivas, de orientación profesional y competencias emprendedoras, las cuales emergen y afloran en esta etapa de desarrollo de los potenciales universitarios. «Una sociedad emprendedora genera una cultura de ofertas empresariales; las economías de consumo, en cambio, ven reducirse en forma gradual el número de personas dispuestas a la innovación, con lo cual se disminuyen necesariamente las plazas de trabajo y se sientan las bases del desempleo estructural». (Enciso, 2010: 69).

En este sentido, las aulas de clase son el escenario natural para el encuentro del emprendimiento como práctica y como conocimiento; los procesos de enseñanza se enriquecen no solo con los nuevos conceptos, sino con aquellos que se originan en otros campos del saber, pero que 
deben ser articulados a la experiencia formativa del estudiante a través de nuevos espacios que configuren el nuevo perfil Emprendedor. Algunos obstáculos para el emprendimiento según Schnarch, 2008, son: «el temor a lo desconocido, el miedo al ridículo, la inseguridad, el deseo de triunfar fácil, prejuicios, o incluso la baja autoestima, además de simplemente no pensar».

Se realizó un acercamiento con los estudiantes y los actores sociales que intervienen como formadores y/o potencializadores de las habilidades emprendedoras juveniles, para identificar el estado de la situación actual frente a temas de innovación y emprendimiento. El estudio se justifica en la falta de oportunidades de empleo, desaprovechamiento de los recursos destinados al emprendimiento y en la deficiencia de la cátedra empresarial desarrollada en las instituciones educativas objeto de estudio. Todo comienza cuando las infraestructuras de información y los sistemas educativos logran posibilidades eficientes que permiten acelerar el aprendizaje colectivo. (Pereira, 2011: 133).

Para cumplir con el objetivo de la investigación, se realizaron encuentros, talleres y encuestas para estudiantes, por otra parte, se consulta con el personal docente encargado de la cátedra empresarial, para conocer sus apreciaciones frente a la aplicación de la normatividad vigente de la temática objeto de estudio.

Desde el punto de vista social, se podría afirmar que el desarrollo de nuevas empresas e ideas de negocio, es una acción muy importante y representativa, vital para aliviar la pobreza y convertir a los estudiantes en agentes productivos e involucrarlos como potenciales emprendedores en la economía local y regional.

Ahora, el emprendimiento empresarial desde la formación académica, representa la posibilidad de que la sociedad en general pueda acceder progresivamente a nuevas etapas de desarrollo.
La cultura de crear empresa y ser emprendedor es decisiva; ya no es un fenómeno reducido sino que es: un concepto que tienen que incorporar e interiorizar a todos los bachilleres para elaborar un mejor proyecto de vida. Podría catalogarse la asignatura de Creatividad Empresarial como un espacio de innovación educativa, es decir, como una herramienta de transformación asociada con la calidad y la competitividad de las personas. En este sentido, la innovación, desde una perspectiva social, "significa asumir el desafío de...formar ciudadanos productivos y competitivos, para un mundo en permanente cambio" (Blanco y Messina, 2000, p. 42)". (Tarapuez, 2008: 33).

Y es allí, en donde el papel desempeñado por los actores que participan en el desarrollo de un «perfil emprendedor», (Dirube, 2011: 224) en el impulso de nuevas ideas, en la creatividad y sobre todo en la capacidad de saber descubrir las potencialidades de futuros empresarios de la región, juega el verdadero rol y la importancia para poder potencializar el emprendimiento local y mitigar algo de la problemática actual de los jóvenes bachilleres.

\section{Antecedentes del emprendimiento en pasto}

El objetivo general de la investigación es diagnosticar la situación actual del Emprendimiento como instrumento para la elaboración de una propuesta que facilite la implementación de la cátedra de emprendimiento en las Instituciones Educativas de la ciudad de Pasto.

Para lograr el objetivo se procedió a analizar los principales factores que influyen en el desarrollo del perfil emprendedor de los estudiantes de grados décimo y once de las instituciones educativas objeto de estudio. Se realizó un estudio del entorno y de los distintos actores sociales identificando como influyen en el desarrollo del 
potencial emprendedor de los estudiantes objeto de estudio. Posteriormente se identifican alternativas de mejoramiento para la implementación de la cátedra de emprendimiento, que permita incentivar y promover el espíritu emprendedor en los estudiantes de grado décimo y once de las Instituciones Educativas objeto de estudio según lo dispuesto en la Ley 1014 de 2006.

El entorno en el cual se está desarrollando la cultura emprendedora de los jóvenes estudiantes de la ciudad de Pasto no es el mejor; al hacer un trabajo de campo con la población estudiantil de grados décimo y once de los distintos colegios e instituciones educativas de la ciudad, se evidencia que la mayoría de los estudiantes tienen poca claridad en cuanto a qué es el Emprendimiento, no identifican o no relacionan adecuadamente este término y no saben cómo, dónde o con quien se puede crear empresa. Por otro lado, la gran mayoría de las instituciones educativas no han implementado el programa de emprendimiento para la «formación de cultura emprendedora» (Briones, 2011: 7) o lo han hecho por cumplir con la normatividad vigente, problemática que es más evidente en las instituciones de carácter público; muchos establecimientos no tienen siquiera en sus planes implementar este programa, por otra parte el $44.09 \%$ de los estudiantes de grados 10 y 11 están concentrados en 7 instituciones educativas (INEM Luis Delfín Insuasty Rodríguez - Colegio Ciudad de Pasto - Institución Educativa Municipal Libertad - Institución Educativa Municipal Técnico Industrial - Liceo Central de Nariño - Colegio María Goretti - Escuela Normal Nacional) y que a su vez conforman el $60.67 \%$ de los estudiantes matriculados en grados 10 y 11 de los establecimientos educativos públicos; este es realmente un porcentaje muy importante de la población estudiantil de la ciudad de Pasto.

Por otro lado, hay un interés especial en esta investigación el cual surge a raíz de la situación económica actual, que ha promovido la búsqueda de alternativas de trabajo, y que obliga a fomentar en los nariñenses y pastusos su espíritu emprendedor y de formación para crear alternativas de actividad empresarial que generen empleo en lugar de demandar trabajo. Shapero afirma que cada evento emprendedor está denotado por tomar la iniciativa, reunir recursos, gerenciamiento, relativa autonomía y tomar riesgos (Citado por Poncio, 2010: 22).

El emprendimiento se ha establecido en los últimos años como la base del desarrollo y crecimiento de un país debido a que trae consigo nuevas ideas y más empresas productivas que le aportan una dinámica a la sociedad. Aun así, es de vital importancia entender que el emprendimiento debe ir obligatoriamente ligado a la educación y debe ser desarrollado en todos los ámbitos, tanto en el entorno social como en el personal, para que el estudiante tenga la oportunidad de desarrollar sus habilidades emprendedoras y consolidar su idea de negocio (Ramos, 2012: 30).

El objetivo es lograr, que el tema de emprendimiento sea considerado como un elemento estratégico en el mejoramiento continuo de la ciudad de Pasto y de esta forma aprovechar cada uno de los beneficios que traen consigo las tendencias del desarrollo. Por ello, esta investigación se orientó a estudiar el caso de la población que es llamada el futuro y esperanza económica colombiana, los estudiantes de últimos grados de básica secundaria, caracterizándolos, analizando su entorno e identificando riesgos y obstáculos con el ánimo de aprovechar sus capacidades y potencialidades para que finalmente sean identificados aquellos individuos e instituciones que han desarrollado o interiorizado al Emprendimiento como proyecto de Vida. El papel clave para lograr este proceso está en manos de las instituciones educativas quienes tienen bajo su responsabilidad la preparación de alumnos capaces de enfrentarse y competir con un mercado laboral demasiado competitivo, logrando utilizar 
las herramientas dadas para aportar al desarrollo económico y social del país.

\section{Metodología}

El método de investigación es, según Carlos $E$. Méndez «El procedimiento riguroso, formulado de una manera lógica, que el investigador debe seguir en la adquisición del conocimiento». Esta es una investigación de corte cualitativo ya que se hace uso de un método de investigación usado principalmente en las ciencias sociales que se basa en cortes metodológicos basados en principios teóricos tales como la fenomenología y la interacción social empleando métodos de recolección de datos que son no cuantitativos, con el propósito de explorar las relaciones sociales y describir la realidad tal como la experimentan los correspondientes. Es además de tipo analítico y descriptivo; analítico porque se hace un estudio pormenorizado de cada uno de los componentes o características de la población objeto hacia una realidad específica; y descriptivo ya que se describen los datos y características de la población o fenómeno en estudio. «El cuestionario más utilizado para medir la innovación en los emprendedores es KAll (KirtonAdaptation-InnovationInventory) de Kirton(1994)» (Citado por Sánchez y Sánchez, 2005: 137).

\section{Muestra}

Para efectos de la recolección de la información, se tomó de la población mencionada anteriormente las opiniones en una muestra aleatoria de 233 estudiantes de décimo y once grado de cada una de las instituciones educativas; utilizando el método aleatorio simple teniendo en cuenta que este número de estudiantes representa el nivel de confianza necesario para el estudio. Las 7 instituciones educativas de Pasto que albergan el grueso de la población estudiantil según información suministrada por la secretaria municipal de educación y verificada con los directivos de cada IEM tienen matriculado 4370 estudiantes. Se calcula una muestra para un universo finito, utilizando el muestreo aleatorio simple.

Los instrumentos de recolección de la información que se utilizaron fueron la encuesta, la entrevista personalizada y el sondeo de opinión. Es importante destacar que los criterios para seleccionar los expertos en el tema de emprendimiento, están fundamentados en la experiencia que, cada uno de ellos ha adquirido desde su vivencia, participación en la institución educativa o a través de los diferentes cargos que han desempeñado en el transcurso vida profesional.

\section{Resultados y discusión}

La palabra emprendimiento proviene del francés «entrepreneur» (Cuervo y otros, 2007) referente a «capacidad de una persona para hacer un esfuerzo adicional por alcanzar una meta u objetivo».

Según el observatorio del emprendimiento de Bogotá. (2007) «una persona emprendedora es flexible, dinámica, capaz de asumir riesgos, innovadora, creativa y orientada al crecimiento». En otras palabras, el emprendimiento es una actitud propia de cada persona que le da la capacidad y la motivación para emprender nuevos proyectos que le permitan generar beneficios, tanto personales como sociales.

Esta actitud se convierte en un motor que permite avanzar, con mucha perseverancia y sacrificio personal, hacia el cumplimiento de las metas y hacia terrenos de innovación alcanzando mayores logros. «Una manera de pensar y actuar orientada hacia la creación de riqueza. Es una forma de pensar, razonar y actuar centrada en las oportunidades, planteada con visión global y llevada a cabo mediante un liderazgo equilibrado y la gestión de un riesgo calculado, su resultado es la creación de valor que beneficia a la empresa, la economía y la sociedad» (Varela, 2008). 
Actualmente, el emprendimiento ha cobrado una gran importancia debido a los constantes problemas económicos que viven las sociedades. Los seres humanos buscan independencia y estabilidad económica por medio de proyectos propios, para no hacer parte de los altos índices de desempleo que viven países como Colombia. Todo se basa en un cambio de mentalidad que busca pasar de empleado a empleador por medio de llevar a cabo ideas de negocio competitivas en el mercado. La mentalidad emprendedora surge de una actitud de insatisfacción por el mejoramiento de determinadas situaciones de estabilidad que le pueda generar un trabajo como asalariado. Es importante entender que en países en vía de desarrollo, en los cuales los estados no tienen la capacidad de subsidiar el desempleo, la mejor alternativa para garantizar el acceso a los recursos que satisfagan las necesidades básicas de una persona y la realización de esta, es tratar de convertir al asalariado en empresario. Es por esta razón que los gobiernos han destinado un presupuesto para apoyar a las personas emprendedoras que con su actitud y creatividad puedan generar resultados generando empleos que sean capaces de contribuir al mejoramiento de la calidad de vida en general.

Para crecer económicamente y mejorar la calidad de vida de las personas, es necesario crear una cultura de emprendimiento que motive a las personas a vencer las resistencias establecidas por el comportamiento económico y social global, y el espacio y el tiempo ideal es en las aulas de clase y desde temprana edad. Las sociedades y economías que promueven el desarrollo del emprendimiento y la innovación en sus miembros, logran mayor productividad y se vuelven más competitivas y seguras en el mercado globalizado. Para el observatorio del emprendimiento (2009) «En las políticas locales, el emprendimiento se consolida como estrategia para lograr mayor desarrollo económico, fomentar la creación de empresas competitivas y sostenibles, consolidar nuevos proyectos productivos, incrementar las exportaciones, y crear nuevos empleos en condiciones de estabilidad y formalidad, con resultados positivos en la calidad de vida de los habitantes».

Según el DANE (2005) en Colombia, el 20\% de la población son jóvenes entre los 15 y 25 años, de los cuales: el $49.18 \%$ están matriculados en educación secundaria, el $32.9 \%$ de los bachilleres pasan a algún tipo de educación superior y un $22.6 \%$ se considera población juvenil desempleada.

Por tanto, se procede a identificar y caracterizar el perfil emprendedor de los estudiantes de grado 10 y 11 de las instituciones educativas de la ciudad de Pasto objeto de estudio, cuáles son sus características más relevantes, conocer que actitudes, aptitudes y competencias definen el Espíritu Emprendedor en los estudiantes, identificar la percepción que tienen al respecto del emprendimiento en sus Instituciones Educativas y finalmente distinguir que motiva al emprendimiento como una herramienta para formar competencias laborales en esta población, teniendo en cuenta que «El autoempleo y la creación de micro y pequeñas empresas, es uno de los caminos que los jóvenes pueden explorar para forjar su futuro».

Para ello, se estudian las características de emprendimiento que pueden ser orientadas desde la educación secundaria, el impacto de la educación académica recibida (la cátedra de emprendimiento) y como la familia, el entorno y los orientadores han influido en estos procesos de formación, Los resultados fueron los siguientes:

Los estudiantes de grados 10 y 11 de ese periodo, la mayoría habían escuchado hablar de emprendimiento pero pocos tenían un conocimiento claro sobre en qué consiste y para que les puede servir en el desarrollo de su proyecto de vida. 
Los Colegios e Instituciones educativas de la Ciudad no se habían preocupado lo suficiente por implementar la asignatura de emprendimiento o alguna asignatura afín que empiece a moldear una actitud emprendedora juvenil; problemática que se agudiza en las instituciones públicas.

Se evidencia que la mayoría de los estudiantes encuestados han escuchado hablar de emprendimiento y conocen algo de la temática al respecto, haciendo referencia al $89.78 \%$ de los estudiantes, lo que significa que es usual el tema para ellos.

Cabe resaltar que el papel desempeñado por el Servicio Nacional de Aprendizaje SENA a través del «Fondo Emprender FE» (SENA, 2008), de los "club de emprendedores» de algunas empresas e instituciones académicas y por la importancia que le ha brindado el actual gobierno a este tema, hacen que este tema no sea desconocido en su totalidad por los estudiantes de los colegios.

Pero el hecho de que hayan escuchado hablar de emprendimiento no significa que sepan que es y para que les sirve en el desarrollo de su proyecto de vida o como se pueden beneficiar en un futuro cercano.

A continuación se presenta el estado de avance que presentan los colegios investigados frente al tema de emprendimiento:

Tabla 1. Cátedra de Emprendimiento

\begin{tabular}{|c|c|c|}
\hline $\begin{array}{c}\text { Cátedra de } \\
\text { Emprendimiento IEM }\end{array}$ & Estado & Observación \\
\hline $\begin{array}{l}\text { INEM Luis Delfín Insuasty } \\
\text { Rodríguez }\end{array}$ & $\begin{array}{l}\text { Implementada y funcionado } \\
\text { con óptimos resultados }\end{array}$ & $\begin{array}{l}\text { Se instruye desde el grado noveno } \\
\text { (Bachillerato técnico) }\end{array}$ \\
\hline IEM Ciudad de Pasto & No implementada & Orientación netamente académica \\
\hline $\begin{array}{l}\text { Institución Educativa } \\
\text { Municipal Libertad }\end{array}$ & $\begin{array}{l}\text { Implementada y funcionado } \\
\text { con buenos resultados }\end{array}$ & $\begin{array}{l}\text { Se orienta a los estudiantes desde } \\
\text { el grado } 10 \text { (excepto a bachillerato } \\
\text { académico }(30 \%)\end{array}$ \\
\hline $\begin{array}{l}\text { Institución Educativa } \\
\text { Municipal Técnico Industrial }\end{array}$ & $\begin{array}{l}\text { Implementada y funcionado } \\
\text { con óptimos resultados }\end{array}$ & $\begin{array}{l}\text { Se orienta a los estudiantes desde } \\
\text { el grado } 10 \text { pero se quiere suprimir } \\
\text { para reforzar lo académico }\end{array}$ \\
\hline IEM María Goretti & No implementada & $\begin{array}{l}\text { Pero se maneja la metodología de } \\
\text { la transversalidad aportando a la } \\
\text { creatividad y la investigación }\end{array}$ \\
\hline Escuela Normal Nacional & No implementada & Orientación Normalista \\
\hline IEM Liceo Central de Nariño & $\begin{array}{l}\text { Implementada y funcionado } \\
\text { con buenos resultados }\end{array}$ & $\begin{array}{l}\text { Se forma a los estudiantes desde } \\
\text { el grado } 9 \text {, se está iniciando con } \\
\text { la implementación y temática } \\
\text { de estudio }\end{array}$ \\
\hline
\end{tabular}

Fuente: Esta investigación.

El resultado obtenido con relación al plan o proyecto de vida que tienen los estudiantes encuestados es interesante, el $74.9 \%$ de ellos se imaginan o proyectan viviendo, trabajando, estudiando o realizando otra actividad fuera de la ciudad de Pasto. Desde el punto de vista de la cultura emprendedora y la «capacidad del emprendedor» (Varela, 2008) para visionarse y proyectarse metas altas es un valor muy positivo. Pero si lo tomamos como aquella única posibilidad que ven los estudiantes para tener un buen futuro y que si quieren algo bueno no lo encontraran en Pasto, el resultado no es bueno para los intereses regionales. 
Al investigar sobre el perfil emprendedor, se pudo percibir el tipo de ideología del individuo y como el mismo se caracteriza; según la cultura del emprendimiento y el perfil emprendedor, un individuo debe considerarse creativo o sentirse líder e interiorizar estos atributos por encima de otras características. Un tercio de los entrevistados (33.03\%) se considera trabajador más que líder o creativo, lo que evidencia que este grupo de personas no tienen claramente definida una cultura emprendedora y el $84 \%$ manifiesta que en el mediano o largo plazo probablemente no crearan empresa sino que serán empleados. El $46.17 \%$ de los estudiantes encuestados se identifican con el liderazgo o la creatividad, que son atributos de la cultura emprendedora; esta la población que se convierte en la verdadera esperanza emprendedora de la región, son casi la mitad de los estudiantes quienes pueden con el apoyo apropiado y con la orientación correcta crear empresa en el futuro cercano; este es un grupo de personas que son capaces de asumir riesgos y de liderar proyectos creativos e innovadores. Finalmente un quinto de la población objeto, que es un caudal relativamente alto teniendo en cuenta la cantidad de estudiantes de la ciudad; tienen un pensamiento inmaduro con respecto a su futuro personal y/o profesional, o no le han dado la importancia necesaria a este tema o a su futuro cercano, este grupo de personas quizás no tengan bases fuertes con respecto a la cultura emprendedora.

\section{Conclusiones}

Se logró establecer que un tercio de los entrevistados (33.03\%) se considera trabajador más que líder o creativo, lo que evidencia que este grupo de personas no tienen claramente definida una cultura emprendedora y que en el mediano o largo plazo probablemente no crearan empresa sino que serán empleados. El $46.17 \%$ de los estudiantes encuestados se identifican con el liderazgo o la creatividad, que son atributos de la cultura emprendedo- ra; esta la población que se convierte en la verdadera esperanza emprendedora de la región, quienes pueden con el apoyo apropiado y con la orientación correcta crear empresa en el futuro cercano; este es un grupo de personas que son capaces de asumir riesgos y de liderar proyectos creativos e innovadores. Finalmente un quinto de la población objeto, que es un caudal relativamente alto teniendo en cuenta la cantidad de estudiantes de la ciudad; tienen un pensamiento inmaduro con respecto a su futuro personal y/o profesional, o no le han dado la importancia necesaria a este tema o a su futuro cercano, este grupo de personas quizás no tengan bases fuertes con respecto a la cultura emprendedora.

La formación emprendedora no puede existir sin creatividad. Los emprendedores crean constantemente nuevas fórmulas para resolver sus problemas, un emprendedor estimula su propio ambiente creativo, pensando siempre en términos de soluciones y oportunidades, más que viendo los acontecimientos como un problema.

Se concluye que para fomentar la cultura del emprendimiento es preciso que se inicie un proceso desde los primeros años de vida de las personas. Este proceso debe contemplar estrategias encaminadas a llevar a la persona al convencimiento que mediante la creación de proyectos productivos se puede a llegar a triunfar tanto personal como económicamente. Lamentablemente nuestro sistema educativo se ha enfocado a formar empleados y asalariados más no empresarios, un aspecto importante es que los directores y educadores reconocer la necesidad de fomentar la cultura emprendedora.

Además del sistema educativo, también la misma estructura familiar y social llevan a las personas a ser empleados y no emprendedores. La cultura del emprendimiento debe ser una política y un compromiso de estado, como lo es la educación en general. Afortunadamente, hoy se 
cuenta con el marco normativo en la ley 1014 de 2006 (enero 26) o Ley de fomento a la cultura del emprendimiento, que sienta las bases para la realización de planes, programas y currículos que propendan por la consolidación de competencias emprendedoras en los estudiantes desde temprana edad.

El emprendimiento debe estar dentro de los programas educativos de la población, desde los primeros años hasta la educación superior. Es una de las formas más efectivas de propiciar desarrollo para una comunidad. La presente investigación está orientada hacia los colegios por tratarse de una orientación social que se enmarca en el direccionamiento estratégico de la UNAD, ya que dentro de las muchas clases de emprendimiento, la principal es la que se desarrolla por necesidad, dada las características de un gran porcentaje de la población objeto de estudio. El emprendimiento social orienta los negocios no solamente al lucro, sino también a la conciencia de ser socialmente responsable con la sociedad que le rodea, orientando los procesos a la consecución de la calidad de vida de las comunidades.

Las personas desde su más temprana edad, deben ser formadas con ese espíritu emprendedor que les permita en un futuro ser empresarios, generadores de riqueza y de empleo. El sistema educativo tiene como objetivo el formar las mentes y las habilidades de la población, es por ello que se debe incluir en el desarrollo de competencias. Todas aquellas que tienen que ver con el emprendimiento y la formación de la población con una mentalidad emprendedora, empresarial. Es posible que los pequeños eventos no lleven directamente a la creación de empresa pero si siembran la semilla de futuros emprendimientos. Las ferias de emprendimiento, las ideas de negocio, las clínicas de ventas, ruedas de negocios, entre otros contribuyen con la consolidación futura del emprendimiento.
Históricamente, el sistema educativo ha sido diseñado para formar asalariados, empleados, pero no para formar empresarios, generadores de riqueza y bienestar social. Para contribuir a dar soluciones efectivas es importante la articulación de acciones desde el estado, las empresas y la academia, con un compromiso que permita el diseño y la implementación de las estrategias encaminadas a fomentar la cultura del emprendimiento en la población.

Todas las universidades deben intervenir y darle esa importancia a este tema, por ello la UNAD se ha comprometido con el emprendimiento, en este momento se encuentra desarrollando espacios con los colegios denominados VIVE la UNAD, la cual se creó con el fin de promocionar los programas de educación superior, pero uno de sus componentes es realizar talleres de emprendimiento con gran acogida por los estudiantes de grados 10 y 11 y donde se detecta la gran necesidad de continuar en el trabajo de formación para el emprendimiento.

\section{Recomendaciones}

El estudiante necesita apoyo para sacar a flote todo el potencial que él tiene ya que su creatividad es ilimitada y solo necesitan de disponer de espacios para concretar sus sueños e ideas.

Los estudiantes se incentivan cuando al finalizar un proyecto pueden obtener un beneficio personal de él; los estudiantes son en su mayoría de escasos recursos económicos y ellos tienen la posibilidad de fabricar prototipos como parte de su proyecto, y el hecho de poderlo utilizar es el mejor estimulo para continuar aportando a su entorno, por medio de soluciones a los problemas a nivel práctico, es un incentivo de tipo emocional, espiritual, más que económico.

No se puede dar de lo que no se tiene, un profesor no puede enseñar emprendimiento si el mismo no ha sido emprendedor en su proyecto 
de vida, sería muy importante que personajes emprendedores de la región den a conocer su experiencia a los jóvenes estudiantes para ver que han hecho que los hizo diferentes; el problema está en que muchos de los que enseñan estos nuevos conocimientos no interiorizan lo enseñado en sus vidas.

Algunas instituciones educativas forman a sus estudiantes para ingresar a una universidad, pero no todos tienen acceso a esta posibilidad, por tanto es muy conveniente crear aptitudes emprendedoras; si no hay posibilidades de implementar en el corto plazo una cátedra de emprendimiento, por lo menos hacer acciones que se encaminen hacia ese norte. El poseer una actitud positiva y creer que si se pueden hacer las cosas son características que debe poseer un emprendedor; por otra parte no tienen el espacio para emprender, para ganar experiencia en este campo, y por otro lado el ente gubernamental no apoya lo suficiente a los estudiantes especialmente económicamente (créditos).

La UNAD ha venido evolucionando de manera significativa en la participación para formación y apoyo de actividades emprendedoras, tales como orientación a proyectos de negocio, desarrollo de empresas de familia y asesoría en la incursión de las diferentes empresas al mercado local, nacional y global, dentro de sus acciones de proyección social, ha involucrado a los docentes, los alumnos y en general a toda la comunidad, para participar en diferentes grupos asesores y consultores, los cuales brindan alternativas estructuradas y sustentadas para ponerlas al servicio de todas las personas interesadas en crear nuevas empresa y nuevos proyectos.

\section{Referencias}

Briones, Jorge Luis. (2011). Google: Tomo 6. Empresas ganadoras. En: Emérgia. Generando emprendedores para el siglo XXI. España: B - Prodetur, 2011. p 7.http:// site.ebrary.com/lib/unadsp/Doc?id=10458292\&ppg=8

Cuervo Alvaro.; Ribeiro Domingo; Roig Salvador. (2007): Entrepreneurship: Concepts, Theory and Practice. En: Cuervo, A.; Ribeiro, D.; Roig, S. (Eds): Entrepreneurship: Concepts, Theory and Perspective. pp. 1-20. Springer, New York Bogota: Uniandes Libros Virtuales.

Dirube José Luis. (2011). ¡Quiero ser empresario!. Madrid: Ediciones Díaz de Santos. p 224. http://site.ebrary. com/lib/unadsp/Doc?id=10527010\&ppg=224

Enciso Congote .Juan David. (2010). El emprendimiento y el bien común: ¿competencias complementarias o excluyentes?.Educación y Educadores, 2010, Volumen 13 ( 1): 63-76. http://site.ebrary.com/lib/unadsp/ Doc id $=10565893 \&$ ppg $=8$.

Ley 1014 (2006). Ley de fomento a la cultura del emprendimiento. República de Colombia.

Méndez Carlos. (1995). Metodología. Guía para elaborar diseños de investigación en ciencias económicas, contables y administrativas. 2ed. Santafé de Bogotá: McGraw-Hill - Interamericana S.A.

Murcia Cabra Héctor. (2011). Creatividad e innovación para el desarrollo empresarial.Colombia: Ediciones de la U, 2011. p 16. http://site.ebrary.com/lib/unadsp/ Doc? $i d=10559595 \& p p g=19$

Observatorio de Emprendimiento Cámara de Comercio de Bogotá. Edición 1 septiembre de 2007 - 2009.

Pereira Fernando; Osorio Fabián; Medina Lina María. (s.f.). Emprendimiento y sus implicaciones en el desarrollo de Santiago de Cali 2010 - 2011: una perspectiva basada en GEM.Colombia: Ediciones de la U, 2011. p 131. http://site.ebrary.com/lib/unadsp/ Doc? $\mathrm{id}=10559736 \& p p g=133$

Poncio Dario Alberto. .(2010). Animarse a emprender. Argentina: Eduvim - Editorial Universitaria Villa María, 2010. p 22. http://site.ebrary.com/lib/unadsp/ Doc? $\mathrm{id}=10536202 \& p p g=23$

Ramos Ruiz José Luis; Moreno Cuello; Gómez Leivis. (2012). Incubadoras de empresas en Colombia: balance y recomendaciones de políticas.Colombia: Universidad del Norte, 2012. p 30. http://site.ebrary.com/lib/ unadsp/Doc? $i d=10584324 \& p p g=30$ 
Sánchez, Almagro Maria Luisa; Sánchez Herrero Silvia A. (2005). Perfil psicológico del autoempleado.España: Universidad Complutense de Madrid, 2005. p 137. http:// site.ebrary.com/lib/unadsp/Doc?id=10101994\&ppg=137

Schnarch Kirberg Alejandro. (2008). Creatividad aplicada: cómo estimular y desarrollar la creatividad a nivel personal, grupa y empresarial (2a. ed.). Colombia: Ecoe Ediciones, http://site.ebrary.com/lib/unadsp/ Doc? $\mathrm{id}=10552963 \& \mathrm{ppg}=65$

SENA (2008). Apoyamos proyectos productivos, Fondo Emprender, Bogotá: Servicio Nacional de Aprendizaje. http://www.fondoemprender.com/BancoConocimiento/F/ Fondo_Emprender/Fondo_Emprender.ASP

Tarapuez Chamorro Edwin; Lima Rivera Carolina. (2008). Creatividad empresarial: elementos teóricos e instrumentos didácticos. Colombia: Ecoe Ediciones. http://site. ebrary.com/lib/unadsp/Doc id=10552533\&ppg=33.

Varela Rodrigo. (2008), Innovación Empresarial, Arte y Ciencia en la Creación de Empresas. Pearson Educación de Colombia S.A., Segunda edición. Bogotá, CoIombia. Observatorio de Emprendimiento Cámara de Comercio de Bogota Edición 1 Septiembre de 2007 2009.

Varela Rodrigo. (2008). La Universidad y su Función en el Desarrollo Empresarial Latinoamericano. Revista Javeriana, Edición 743. 